\title{
A NONLINEAR STEADY STATE TEMPERATURE PROBLEM FOR A SEMI-INFINITE SLAB
}

\author{
DANG DINH ANG
}

We propose to investigate the following boundary value problem:

(1) $w_{x x}+w_{y y}=0,0<x<\pi, 0<y<\infty$,

(2) $w(0, y)=f_{1}(y), 0 \leqq y<\infty$,

(3) $w(\pi, y)=f_{2}(y), 0 \leqq y<\infty$,

(4) $-w_{y}(x, 0)=G[x ; w(x, 0)], 0<x<\pi$,

where $f_{1}, f_{2}$ are real-valued continuous functions on [0, [ vanishing at infinity, and $G$ is a nonlinear real-valued function of two real variables satisfying the following conditions:

$G[x ; u]$ is strictly decreasing in $u, G[x ; c]=0$ where $c$ is a constant such that

(5) $c \geqq \sup \sup _{x}\left(f_{1}(x), f_{2}(x)\right)$,

(6) $G[x ; u]$ is continuous in $x$ and $u$ jointly.

Physically, $w$ is the temperature, and the condition (4) with $G$ satisfying (5) and (6) is a generalization of Newton's law of cooling; $-w_{y}(x, 0)=l\left(c_{0}-w(x, 0)\right), l$ and $c_{0}$ being constants.

Mann and Blackburn [1] investigated a particular case of the foregoing problem corresponding to $f_{1}=0=f_{2}, c=1$, and $G$ independent of the first argument $x$. The reason we consider this problem again is two-fold: first, the argument given in [1] in the proof of the existence theorem (loc. cit. Theorem 3) does not directly carry over to the present more general case, and second, we wish to present a method for constructing the solution in certain particular cases, whereas the authors in [1] were more concerned with existence theorems. Our main tool is a fixed point theorem of H. Schaefer [2].

By a solution of the boundary value problem (1)-(6), we mean a function $w$ harmonic in the open strip, satisfying (2)-(6), continuous in $0 \leqq x \leqq \pi, 0 \leqq y<\infty$, and vanishing at infinity. We postulate $w$ to be of the form

(7) $w=w_{1}+w_{2}$, where $w_{1}, w_{2}$ are harmonic in the open strip, and

(8) $w_{1}(0, y)=w_{1}(\pi, y)=0,0 \leqq y<\infty$,

(9) $-w_{1, y}(x, 0)=G[x ; w(x ; 0)], 0<x<\pi$,

(10) $w_{2}(0, y)=f_{1}(y)$,

(11) $w_{2}(\pi, y)=f_{2}(y)$,

(12) $w_{2, y}(x, 0)=0,0<x<\pi$.

By proceeding as in [1], one finds

Received by the editors September 21, 1967. 


$$
\begin{gathered}
w_{1}(x, y)=\frac{1}{\pi} \int_{0}^{\pi} \log \frac{1-2 \exp (-y) \cos (x-z)+\exp (-y)}{1-2 \exp (-y) \cos (x+z)+\exp (-y)} \\
\cdot G[z ; w(z ; 0)] d z, \quad 0<x<\pi, 0<y<\infty .
\end{gathered}
$$

We now seek an integral representation formula for $w_{2}$. We use the technique of Fourier transforms. Our operations with Fourier transforms are purely formal. We are concerned with the final representation formula which will be justified for its sake. Let

(14) $\hat{f}_{1}(w)=\frac{1}{\pi} \int_{0}^{\infty} f_{1}(\zeta) \cos w \zeta d \zeta, \quad \hat{f}_{2}(w)=\frac{1}{\pi} \int_{0}^{\infty} f_{2}(\zeta) \cos w \zeta d \zeta$.

Note that the possibility that these integrals diverge does not concern us. Let

$$
\begin{aligned}
w_{2}(x, y)=\int_{-\infty}^{\infty}(A(w) \exp (i w y-w x) & \\
& +B(w) \exp (i w y-w(\pi-x))) d w .
\end{aligned}
$$

Then by (10) and (11), and formally using the Fourier inversion formula, we get

$$
\begin{aligned}
& w_{2}(0, y)=2 \int_{0}^{\infty} \hat{f}_{1}(w) \cos w y d w \\
& w_{2}(\pi, y)=2 \int_{0}^{\infty} \hat{f}_{2}(w) \cos w y d w .
\end{aligned}
$$

Equating

$$
w_{2}(0, y)=f_{1}(y), \quad w_{2}(\pi, y)=f_{2}(y),
$$

gives

$$
\begin{aligned}
A(w)+B(w) \exp (-w \pi) & =\hat{f}_{1}(w), \\
A(w) \exp (-w \pi)+B(w) & =\hat{f}_{2}(w) .
\end{aligned}
$$

Hence

$$
\begin{aligned}
& A(w)=\Delta(w)\left(\hat{f}_{1}(w)-\hat{f}_{2}(w) \exp (-w \pi)\right), \\
& B(w)=\Delta(w)\left(\hat{f}_{2}(w)-\hat{f}_{1}(w) \exp (-w \pi)\right),
\end{aligned}
$$

where

$$
\Delta(w)=(1-\exp (-2 w \pi))^{-1}
$$


In view of (15), (20), (21), we have

$$
w_{2}(x, y)=\frac{2}{\pi} \int_{0}^{\infty}\left(f_{1}(\zeta) D(x, y ; \zeta)+f_{2}(\zeta) E(x, y ; \zeta)\right) d \zeta
$$

where

$$
\begin{aligned}
D(x, y)= & \int_{0}^{\infty} \Delta(w)(\exp (-w x)-\exp (-2 w \pi+w x)) \\
& \cdot \cos w y \cos w \zeta d \zeta, \\
E(x, y)= & \int_{0}^{\infty} \Delta(w)(\exp (-w(\pi-x))-\exp (-w(\pi+x))) \\
& \cdot \cos w y \cos w \zeta d \zeta .
\end{aligned}
$$

LEMMA 1. (i) $w_{2}$ is harmonic in the open strip.

(ii) $\lim _{y \rightarrow 0} w_{2}(x, y)$ exists for $0 \leqq x \leqq \pi$ and defines a function continuous on $[0, \pi]$.

(iii) $\lim _{y \rightarrow 0} w_{2, y}(x, y)=0,0<x<\pi$.

(iv) $\lim _{x \rightarrow 0} w_{2}(x, y)=f_{1}(y), \lim _{x \rightarrow x} w_{2}(x, y)=f_{2}(y)$.

Proof. The proof of (i), (ii), (iii) is straightforward. The proof of (iv) proceeds by approximating the kernels $D$ and $E$ by the Poisson kernel. Details are omitted.

In view of Lemma 1 and equation (13), the boundary value problem (1)-(6) reduces to the following integral equation

$$
u(x)=\frac{1}{\pi} \int_{0}^{\pi} G[z ; u(z)] K(x, z) d z+w_{2}(x, 0), \quad 0 \leqq x \leqq \pi,
$$

where $u(x)=w_{1}(x, 0)$ and

$$
K(x, z)=\log \frac{1-\cos (x-z)}{1-\cos (x+z)} .
$$

Define

$$
T u(x)=\frac{1}{2 \pi} \int_{0}^{\pi} G[z ; u(z)] K(x, z) d z+w_{2}(x, 0), \quad 0 \leqq x \leqq \pi .
$$

Then $u$ is a solution of (26) if and only if $u$ is a fixed point of $T$. In the sequel, our arguments will be couched in the language of fixed point theorems. Let $E$ be the Banach space of continuous functions on $[0, \pi]$. Then it is clear that $T$ takes $E$ into $E$. It is also clear that $T$ is completely continuous, since the function $\log (1-\cos (x \pm z))$ is integrable in $z$. 
Lemma 2. $T$ has at most one fixed point.

The proof of this lemma proceeds exactly as in the proof of Theorem 4 of [1].

Lemma 3. Let $T_{\lambda}=\lambda T, 0<\lambda \leqq 1$. If $u$ is a fixed point of $T_{\lambda}$, then

$$
\inf _{x} \inf \left(f_{1}(x), f_{2}(x)\right) \leqq u \leqq c .
$$

Proof. Let

$$
\begin{gathered}
v(x, y)=\frac{\lambda}{\pi} \int_{0}^{\pi} \log \frac{1-2 \exp (-y) \cos (x-z)+\exp (-y)}{1-2 \exp (-y) \cos (x+z)+\exp (-y)} \\
\cdot G[z ; w(z, 0)] d z+\lambda w_{2}(x, y) .
\end{gathered}
$$

Then, by Lemma 1 ,

$$
\begin{gathered}
\lim _{x \rightarrow 0} v(x, y)=\lambda f_{1}(y), \\
\lim _{x \rightarrow \pi} v(x, y)=\lambda f_{2}(y), \\
-\lim _{y \rightarrow 0} \frac{\partial v}{\partial y}(x, y)=G[x, u(x)] .
\end{gathered}
$$

To prove the double inequality of the lemma, we use the maximum modulus principle for harmonic functions.

(i) $u$ is $\leqq c$.

Suppose on the contrary that $u(x)>c$ for some $x$. Let $x_{M}$ be such that $u\left(x_{M}\right)=\max u(x)$. Then $G\left[x_{M} ; u\left(x_{M}\right)\right]<0$ by the definition of $G$, since $u\left(x_{M}\right)>c$. On the other hand, $v$ is not constant. Hence, by the maximum princ iple,

$$
-y^{-1}\left(v\left(x_{M}, y\right)-v\left(x_{M}, 0\right)\right)>0, \quad y>0 .
$$

Since $-v_{y}\left(x_{M}, 0\right)=G\left[x_{M}, u\left(x_{M}\right)\right]$, we have a contradiction. This contradiction proves (i).

(ii) $u$ is $\geqq \inf _{x} \inf \left(f_{1}(x), f_{2}(x)\right) \equiv a$.

Suppose on the contrary that for some $x$, we have $u(x)<a$. Let $x_{m}$ be such that $u\left(x_{m}\right) \equiv \inf u(x)$. Then $u\left(x_{m}\right)=\inf v(x, y)$ by the maximum principle. The proof proceeds on the same lines as in part (i).

We now state our existence theorem.

Theorem 1. T has a unique fixed point $u$. Furthermore, $a \leqq u \leqq c$.

Proof. Suppose that $T$ has no fixed point. Then by a theorem of Schaefer [2], there exist a sequence $\left(u_{n}\right)$ of elements of $E$ and a se- 
quence of real numbers $0<\lambda_{n}<1$, such that

$$
u_{n}=\lambda_{n} T u_{n}, \quad\left\|u_{n}\right\| \rightarrow \infty .
$$

But by Lemma $3, a \leqq u_{n} \leqq c$. This contradiction proves that $T$ has a fixed point $u$, which is unique by Lemma 2 . Furthermore, $a \leqq u \leqq c$, by Lemma 3. Thus the theorem is proved.

Theorem 2. The boundary value problem (1)-(6) has a unique solution.

Proof. Each fixed point of the operator $T$ gives rise to a solution of the boundary value problem (1)-(6) in an obvious way. Hence by Theorem 1 , the given boundary value problem has a solution. Let $v(x, y), v^{\prime}(x, y)$ be two solutions of the boundary value problem. Let $w(x, y)=v(x, y)-v^{\prime}(x, y)$. Then $w$ vanishes on the edges $x=0$, $0 \leqq y<\infty$, and $x=\pi, 0 \leqq y<\infty$. Using the maximum modulus principle, and the properties of the function $G[x, u(x)]$, we can prove that $w$ vanishes on the base $0 \leqq x \leqq \pi$. Then, again by the maximum modulus principle, $w$ must be the null function. This proves the theorem.

The problem of actually constructing the solution of the problem is one of great interest. If $T$ is a contraction, i.e., if $\left\|T u-T u^{\prime}\right\|$ $\leqq \alpha\left\|u-u^{\prime}\right\|, 0<\alpha<1$, then the fixed point of $T$ can be obtained by successive approximation. We shall show that even when $T$ is only nonexpansive, i.e., $\left\|T u-T u^{\prime}\right\| \leqq\left\|u-u^{\prime}\right\|$, the fixed point of $T$ can still be obtained by successive approximation.

TheORem 3. Let $T$ be nonexpansive. Then the fixed point $u$ of $T$ can be obtained by successive approximation. More precisely, let $T_{n}=\alpha_{n} T$, $0<\alpha_{n}<1$.

If $\alpha_{n} \rightarrow 1$ and $\alpha_{n}^{n} \rightarrow 0$, then $\lim _{n} T_{n}^{n} w=u$ for any $w$ in $E$.

Proof. By $T_{n}^{n}$ we mean of course the $n$th iterate of $T_{n}$. For each $n$, $T_{n}$ is a contraction, and hence has a fixed point $u_{n}, u_{n}=T_{n} u_{n}$.

By Lemma 3 , the sequence $\left(u_{n}\right)$ is bounded, and, by the above relation, is relatively compact. Hence $\left(u_{n}\right)$ has a cluster value. Now, each cluster value of $\left(u_{n}\right)$ is a fixed point of $T$. Since by Theorem $1, T$ has a unique fixed point, it follows that $\left(u_{n}\right)$ has a unique cluster value $u$. Since $\left(u_{n}\right)$ is relatively compact, $\left(u_{n}\right)$ converges to $u$. Now, if $w$ is any element of $E$,

$$
\begin{aligned}
\left\|T_{n}^{n} w-u_{n}\right\| & =\left\|T_{n}^{n} w-T_{n}^{n} u_{n}\right\| \\
& \leqq \alpha_{n}^{n}\left\|w-u_{n}\right\| .
\end{aligned}
$$

Since $\left(u_{n}\right)$ is bounded, the theorem follows. 
ACKNowledgements. The author had several helpful discussions with Professor Leon Knopoff on the subject matter of this paper.

\section{REFERENCES}

1. W. R. Mann and J. F. Blackburn, A nonlinear steady state temperature problem, Proc. Amer. Math. Soc. 5 (1954), 979-986.

2. H. Schaefer, Über die Methode der a priori-Schranken, Math. Ann. 129 (1955), $415-416$.

UNIVERSITY OF SAIGON AND

University of California, Los Angeles 